\title{
Structure of forecast error covariance in coupled atmosphere-chemistry data assimilation
}

\author{
S. K. Park ${ }^{1,2,3,4}$, S. Lim ${ }^{2,3}$, and M. Zupanski ${ }^{5}$ \\ ${ }^{1}$ Department of Environmental Science and Engineering, Ewha Womans University, Seoul, Republic of Korea \\ ${ }^{2}$ Department of Atmospheric Science and Engineering, Ewha Womans University, Seoul, Republic of Korea \\ ${ }^{3}$ Center for Climate/Environment Change Prediction Research, Ewha Womans University, Seoul, Republic of Korea \\ ${ }^{4}$ Severe Storm Research Center, Ewha Womans University, Seoul, Republic of Korea \\ ${ }^{5}$ Cooperative Institute for Research in the Atmosphere, Colorado State University, Fort Collins, Colorado, USA
}

Correspondence to: S. K. Park (spark@ewha.ac.kr)

Received: 26 October 2014 - Published in Geosci. Model Dev. Discuss.: 10 December 2014

Revised: 14 April 2015 - Accepted: 16 April 2015 - Published: 5 May 2015

\begin{abstract}
In this study, we examined the structure of an ensemble-based coupled atmosphere-chemistry forecast error covariance. The Weather Research and Forecasting (WRF) model coupled with Chemistry (WRF-Chem), a coupled atmosphere-chemistry model, was used to create an ensemble error covariance. The control variable includes both the dynamical and chemistry model variables. A synthetic single observation experiment was designed in order to evaluate the cross-variable components of a coupled error covariance. The results indicate that the coupled error covariance has important cross-variable components that allow a physically meaningful adjustment of all control variables. The additional benefit of the coupled error covariance is that a cross-component impact is allowed; e.g., atmospheric observations can exert an impact on chemistry analysis, and vice versa. Given the realistic structure of ensemble forecast error covariance produced by the WRF-Chem, we anticipate that the ensemble-based coupled atmosphere-chemistry data assimilation will respond similarly to assimilation of real observations.
\end{abstract}

\section{Introduction}

The regional air quality is affected by synoptic weather situations or air masses with special chemical properties (Grell et al., 2000). In prediction of air quality, the coupled physical and chemical processes are essential, which include transport, deposition, emission, chemical transformation, aerosol interactions, photolysis, and radiation (Grell et al., 2005). Optimized initial conditions for a numerical model, including such coupled processes, can be obtained by data assimilation (DA; e.g., Houtekamer and Mitchell, 1998; Eibern and Schmidt, 1999; Wang et al., 2001; Evensen, 2003; Park and Zupanski, 2003; Navon, 2009; Zupanski, 2009). Data assimilation has also been applied to the atmospheric chemical transport models (CTMs) (e.g., Constantinescu et al., 2007; Singh et al., 2011). However, the focus of these studies has not been on developing a fully coupled atmospherechemistry DA system that allows the interaction between atmosphere and chemistry DA components. In typical data assimilation methodologies, such as variational and ensemble, the interaction between different variables is achieved by the forecast error covariance, in particular its cross-variable components. More specifically, the forecast error covariance includes the magnitude and the correlations of forecast errors, showing how the information is spread spatially and among control variables (Singh et al., 2011). Therefore, it is of fundamental interest for the development of atmosphere-chemistry DA to investigate the coupled forecast error covariance. Here, we investigate the structure of the atmosphere-chemistry forecast error covariance using ensemble forecasting, which corresponds to the prediction step of an ensemble data assimilation algorithm (e.g., Zupanski, 2005, 2009). 


\section{Methodology and synoptic case}

In this research, we use the Weather Research and Forecasting (WRF) model coupled with Chemistry (WRF-Chem) as a prediction model (Grell et al., 2005). It simulates the emission, transport, mixing and chemical transformation of trace gases and aerosols simultaneously with meteorology and investigates the regional-scale air quality. Here, the chosen chemistry option is Carbon Bond Mechanism version $\mathrm{Z}$ (CBMZ). More details on the WRF-Chem and the corresponding options used in this study are described in Appendix A.

We chose a synoptic case on 3 September 2005 related to Typhoon Nabi (2005), characterized by an increased impact on the Korean Peninsula. The experiment begins at 00:00 UTC and ends at 06:00 UTC on 3 September 2005. The WRF-Chem is set up with a horizontal resolution of $30 \mathrm{~km}$ and 28 vertical levels. The model domain is centered over the Korean Peninsula, covering an area of approximately $3900 \mathrm{~km} \times 4400 \mathrm{~km}$ with $132 \times 147$ horizontal grid points.

The ensemble forecast includes 32 ensemble members with a $6 \mathrm{~h}$ assimilation window. The initial and lateral boundary conditions for atmospheric variables are provided by the National Center for Environmental Prediction (NCEP) Global Forecasting System (GFS), while those for chemical variables are obtained from climatology. The control variables defined in DA (i.e., variables adjusted during DA) are the WRF-Chem prognostic variables that include dynamical variables such as winds, perturbation potential temperature, perturbation geopotential, water vapor mixing ratio and perturbation dry air mass in the column, and the chemical variables such as ozone $\left(\mathrm{O}_{3}\right)$, nitrates $\left(\mathrm{NO}, \mathrm{NO}_{2}, \mathrm{NO}_{3}\right)$ and sulfur dioxide $\left(\mathrm{SO}_{2}\right)$ as well.

\section{Experimental design}

A common approach to investigating forecast error covariance in data assimilation is to conduct a single observation experiment (Thepaut et al., 1996; Whitaker et al., 2009; Buehner et al., 2010), in which only one observation is assimilated using the full DA system. The analysis increments (i.e., analysis minus guess) from such an experiment show how the observation information is distributed spatially and among different analysis variables (e.g., Buehner, 2005; Singh et al., 2011). However, in order to investigate the structure of a coupled forecast error covariance before real observations are available and even before the full DA algorithm is developed, one can consider the assimilation of a single synthetic observation located at a chosen model grid point. In particular, we define the synthetic observation as

$y_{\text {synth }}=x^{\mathrm{f}}+\sigma_{\mathrm{o}}$,

where $x^{\mathrm{f}}$ is the forecast and $\sigma_{\mathrm{o}}$ is the observation error standard deviation. Following Thepaut et al. (1996, Eq. 3), with some modifications and using Eq. (1), the analysis increment in a single synthetic observation experiment is

$x^{\mathrm{a}}-x^{\mathrm{f}}=\mathbf{P}_{\mathrm{f}}\left(\frac{\sigma_{\mathrm{o}}}{\sigma_{\mathrm{f}}^{2}+\sigma_{\mathrm{o}}^{2}}\right)_{i j k}$,

where $x^{\mathrm{a}}$ is the analysis, $\sigma_{\mathrm{f}}$ is the forecast error standard deviation, and the subscript $i j k$ defines the grid location of the pseudo-observation point. Equation (2) indicates that the analysis increment represents the $i j k$ th column of the forecast error covariance scaled by standard deviations of observation error and forecast error. In our experiments, the forecast error covariance is ensemble-based, as defined in Zupanski (2005) as

$$
\begin{aligned}
& \mathbf{P}_{\mathrm{f}}=\mathbf{P}_{\mathrm{f}}^{1 / 2}\left(\mathbf{P}_{\mathrm{f}}^{1 / 2}\right)^{\mathrm{T}}, \mathbf{P}_{\mathrm{f}}^{1 / 2}=\left(p_{1}^{\mathrm{f}} \ldots p_{N}^{\mathrm{f}}\right), \\
& p_{n}^{\mathrm{f}}=m\left(x_{0}^{n}\right)-m\left(x_{0}\right),
\end{aligned}
$$

where the superscript $\mathrm{T}$ denotes the transpose, the index $n$ refers to ensemble member, $N$ is the total number of ensemble forecasts, $m$ represents the nonlinear WRF-Chem model, and the subscript 0 denotes the initial time of the forecast with corresponding initial conditions $x_{0}$ and ensemble initial conditions $x_{0}^{n}$. In this experiment, the control initial conditions are obtained by interpolation from the NCEP GFS model, while the initial ensemble perturbations are created using the lagged forecast outputs.

Since we are interested in the coupled atmospherechemistry forecast error covariance, we design two experiments with (i) synthetic temperature observation at $250 \mathrm{hPa}$ located at a grid point near $\left(132^{\circ} \mathrm{E}, 23^{\circ} \mathrm{N}\right)$, on the northwestern side of the typhoon, and (ii) synthetic ozone observation at $250 \mathrm{hPa}$ located at a grid point near the eye of the typhoon $\left(134^{\circ} \mathrm{E}, 21^{\circ} \mathrm{N}\right)$.

\section{Results}

We show the impact of single synthetic temperature $(T)$ and ozone $\left(\mathrm{O}_{3}\right)$ observations in terms of the analysis increments $x^{\mathrm{a}}-x^{\mathrm{f}}$ impacting all control variables. As mentioned earlier, our main interest is to examine the cross-variable covariance structure between atmospheric and chemistry variables, since the cross-variable analysis impact is possible only because of the multivariate structure of the coupled ensemble forecast error covariance.

In Fig. 1, we show the impact of synthetic $T$ observation at $250 \mathrm{hPa}$ on the analysis increments of $T, \mathrm{O}_{3}$, nitrogen dioxide $\left(\mathrm{NO}_{2}\right)$, and sulfur dioxide $\left(\mathrm{SO}_{2}\right)$. The analysis increment of $T$ at $250 \mathrm{hPa}$ (e.g., at the same level of synthetic $T$ observation) shows a typical response with nearly circular isolines with the maximum of $0.4 \mathrm{~K}$ at the observation location (Fig. 1a). The analysis increments of $\mathrm{O}_{3}, \mathrm{NO}_{2}$, and $\mathrm{SO}_{2}$ are also shown in vertical cross sections. One can see that $\mathrm{O}_{3}$ (Fig. 1b) and $\mathrm{NO}_{2}$ (Fig. 1c) analyses have the largest 

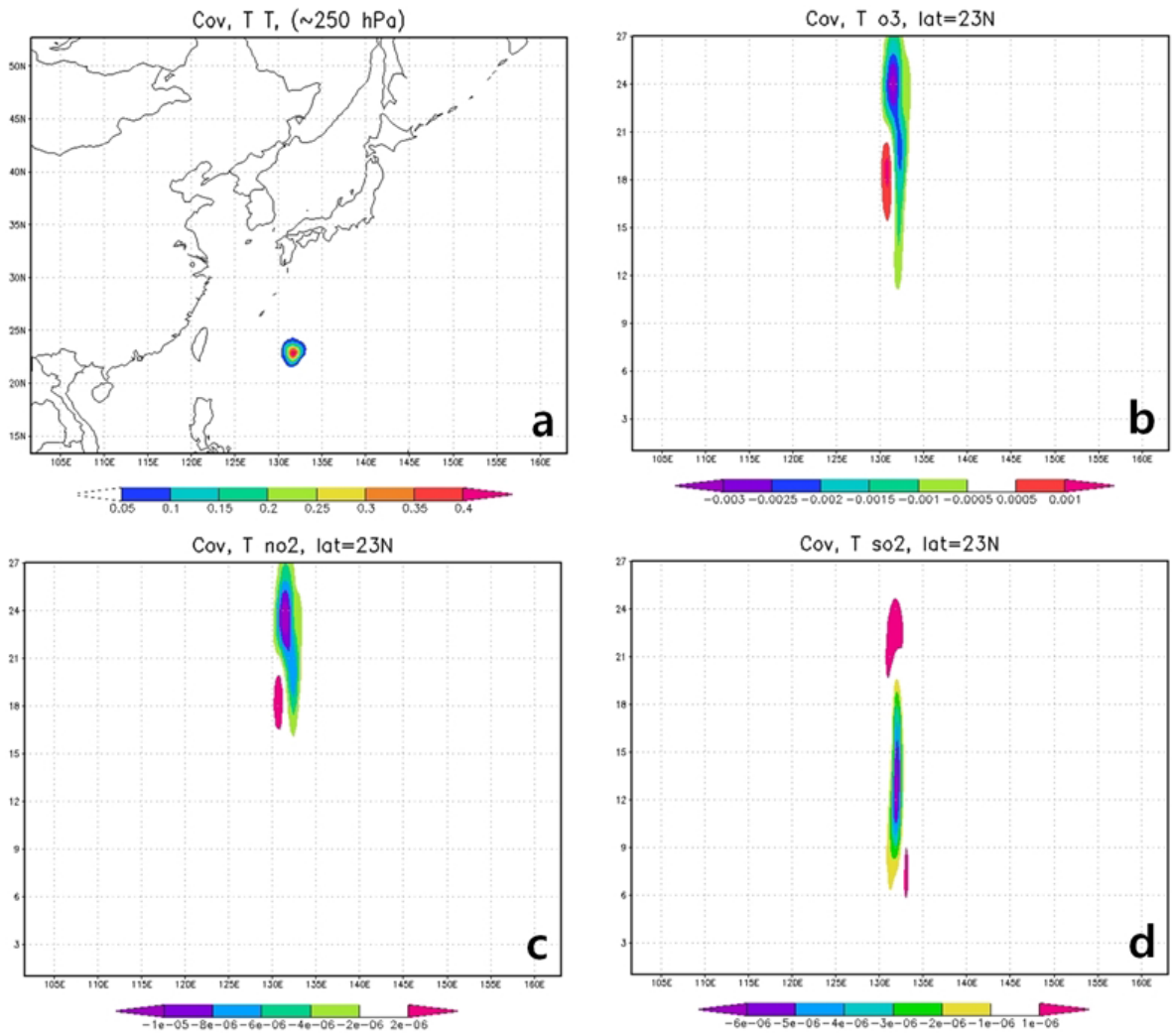

Figure 1. The analysis increments $\left(x^{\mathrm{a}}-x^{\mathrm{f}}\right)$ in response to a single $T$ observation at $250 \mathrm{hPa}$ (near $\sigma$ level 24): (a) horizontal response of $T$ at $250 \mathrm{hPa}$, and vertical responses of (b) $\mathrm{O}_{3}$, (c) $\mathrm{NO}_{2}$ and (d) $\mathrm{SO}_{2}$. In (b)-(d), the vertical axis represents the vertical $\sigma$ levels. Units are ppmv for chemical variables and $\mathrm{K}$ for temperature.

change at the level of single $T$ observation, while the $\mathrm{SO}_{2}$ analysis (Fig. 1d) is mostly impacted near $700 \mathrm{hPa}$ (approximately $\sigma$ level 13). This is likely a consequence of the vertical structure of $\mathrm{O}_{3}$ and $\mathrm{NO}_{2}$, with the largest values in the upper troposphere and the stratosphere, while $\mathrm{SO}_{2}$ typically has the largest values in the lower troposphere (e.g., Meena et al., 2006). The strongest impact of $T$ observation is on $\mathrm{O}_{3}$, with the magnitude up to $0.003 \mathrm{ppmv}$, while the magnitude is somewhat smaller for $\mathrm{NO}_{2}$ and $\mathrm{SO}_{2}$. One can also infer that an increase in $T$ will imply a decrease in $\mathrm{O}_{3}, \mathrm{NO}_{2}$, and $\mathrm{SO}_{2}$. Probably the most important implication of these results is that observations of an atmospheric variable (e.g., temperature) can change the analysis of chemical variables in a physically meaningful way. This means that, even with no chemistry observations in the local area, the analysis of chemical variables can still be adjusted in agreement with standard dynamical variables of the model. On the other hand, if there are chemistry observations in the area, the chemistry analysis change introduced by atmospheric observations will act as an additional dynamical constraint on the final analysis.

In Fig. 2, the impact of $\mathrm{O}_{3}$ single observation at $250 \mathrm{hPa}$ on itself and the other variables is shown. As before, we focus on the vertical cross section of the analysis response. The impact of $\mathrm{O}_{3}$ observation on its own analysis shows the anticipated response, with the largest magnitude at the observation location, approximately $0.02 \mathrm{ppmv}$ (Fig. 2a). Although smaller in magnitude, the analysis increments of $\mathrm{NO}_{2}$ (Fig. 2b) and $\mathrm{SO}_{2}$ (Fig. 2c) show the vertical structure with maxima in the upper and lower troposphere, respectively. It is also notable that an increase in $\mathrm{O}_{3}$ brings about an increase in $\mathrm{NO}_{2}$ and $\mathrm{SO}_{2}$, confirming the direct relationship between these variables as noticed in Fig. 1. The $T$ analysis increment indicates that there is a cooling at the level of $\mathrm{O}_{3}$ observation, while there is a warming above and below (Fig. 2d).

The results shown in Figs. 1 and 2 indirectly confirm that the improved stratospheric ozone distribution by DA can make a better representation of stratospheric temperature and other constituents (e.g., Lahoz et al., 2007).

\section{Conclusions}

The structure of an ensemble-based coupled atmospherechemistry forecast error covariance was examined in the context of the WRF-Chem model. A synthetic single observation experiment was designed in order to evaluate the crossvariable components of the coupled error covariance. Our results indicate that the coupled error covariance has important cross-variable components that allow a physically meaning- 

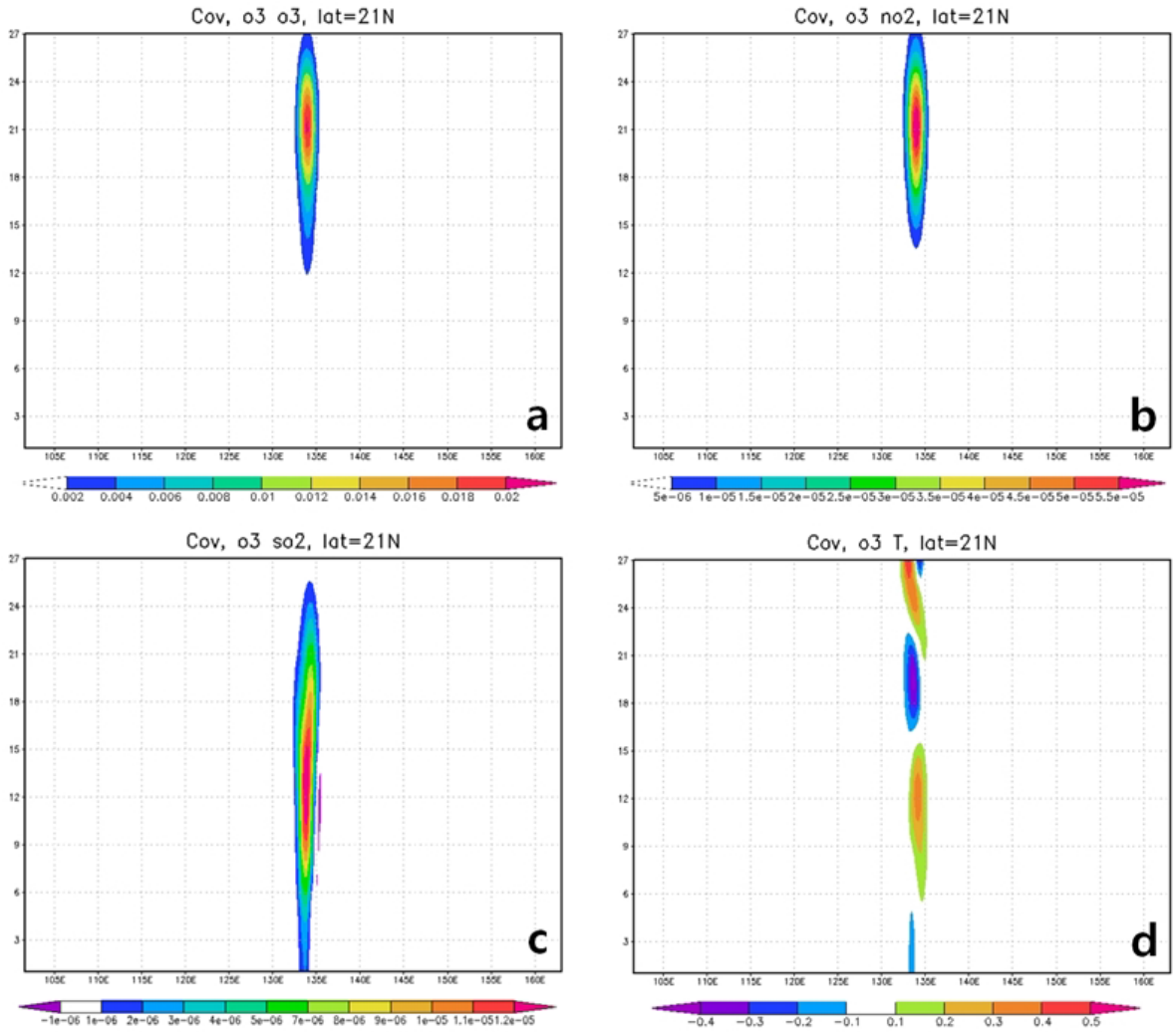

Figure 2. Same as in Fig. 1 but for the vertical cross section of the analysis increments $\left(x^{\mathrm{a}}-x^{\mathrm{f}}\right)$ in response to a single $\mathrm{O}_{3}$ observation at $250 \mathrm{hPa}$ for (a) $\mathrm{O}_{3}$, (b) $\mathrm{NO}_{2}$, (c) $\mathrm{SO}_{2}$ and (d) $T$.

ful adjustment of all control variables, and a much wider impact of observations (e.g., atmospheric observation on chemistry analysis, and vice versa). The analysis increments created in response to synthetic temperature and ozone observations illustrate the complexity of atmosphere-chemistry cross-correlations and the forecast error covariance structure. Given the realistic structure of ensemble forecast error covariance produced by the WRF-Chem, we anticipate that the ensemble-based coupled atmosphere-chemistry data assimi- lation will respond similarly to assimilation of real observations. Therefore, our next step is to apply the WRF-Chem with an ensemble-based data assimilation algorithm (e.g., the maximum likelihood ensemble filter (MLEF); Zupanski, 2005) to assimilation of real chemical and atmospheric observations. 


\section{Appendix A: Description of the WRF-Chem}

In this research, we use the Weather Research and Forecasting (WRF) model coupled with Chemistry (WRF-Chem) version 3.4.1 as a prediction model on a regional scale. As a coupled model, it simulates the emission, transport, mixing and chemical transformation of trace gases and aerosols simultaneously with meteorology using the governing equations with mass and scalar conserving flux form and the terrainfollowing mass vertical coordinate system (Grell et al., 2005; Fast et al., 2006). Therefore, it uses the same transport scheme, horizontal and vertical coordinates, and physics schemes with the same time step (Grell et al., 2005; Fast et al., 2006). Figure A1 represents the flowchart of the WRFChem model. It is made up of the WRF Pre-processing System (WPS), the WRF-Chem model, and the visualization processes. The WPS creates the meteorology data with the terrestrial data and the meteorology initial conditions (ICs) and boundary conditions (BCs), which are provided by the National Center for Environmental Prediction (NCEP) Global Forecasting System (GFS) producing the global latitude/longitude 1 degree resolution and terrestrial data. In the WRF-Chem model, the chemical ICs and BCs are automatically obtained by the climatology. Furthermore, it simulates the evolution of chemical species with the prognostic variables that include both dynamical and chemical variables. For the ensemble experiments, the initial ensemble perturbations are created using the $12 \mathrm{~h}$ lagged forecast outputs. Finally, the Advanced Research WRF post-processing (ARWpost), along with the Grid Analysis and Display System (GrADS), is used for the visualization process.

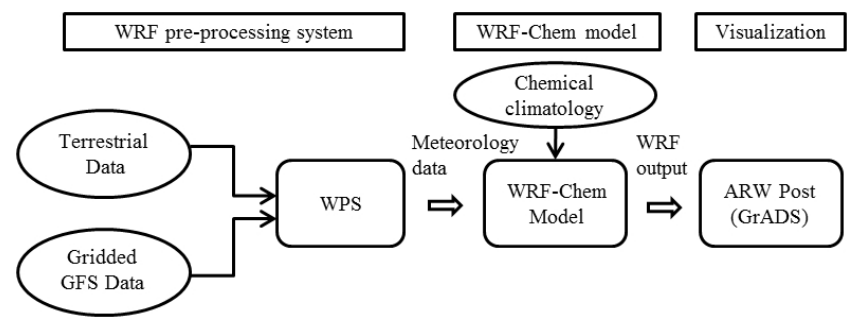

Figure A1. Flowchart of the WRF-Chem model.
We also discuss various physical and chemical processes employed in the WRF-Chem model in more detail. Table A1 summarizes the WRF-Chem configuration options that are used in this study. To evaluate the cross-variable component of forecast error covariance, we select the simplified physical processes rather than sophisticated ones. Regarding the atmospheric processes, we use the recommended physics options for the regional climate case at $30 \mathrm{~km}$ grid size in our experiments. As the chemical options, the Carbon Bond Mechanism version Z (CBM-Z) without a dimethylsulfide scheme is used for the gas-phase chemistry. The CBM-Z photochemical mechanism contains 55 prognostic species and 134 reactions having the lumped structure approach for condensing organic chemical species and reactions (Fast et al., 2006). It also uses a regime-dependent approach based on the partitioned kinetics, such as background, anthropogenic, and biogenic submechanisms for saving the computational time (Fast et al., 2006). Furthermore, we consider the chemical tendency diagnostic for equation budget analysis. However, we did not consider the convective parameterization, which can simulate the subgrid convective transport, wet scavenging, and aqueous chemistry due to a simple experiment setting, even with a typhoon case.

Table A1. Selected WRF-Chem configuration options.

\begin{tabular}{ll}
\hline Atmospheric process & WRF-Chem option \\
\hline Microphysics & WSM 6-class graupel \\
Longwave radiation & CAM \\
Shortwave radiation & CAM \\
Surface layer & Revised MM5 Monin-Obukhov \\
Land surface & Unified Noah LSM \\
Planetary boundary layer & YSU \\
Cumulus parameterization & Kain-Fritsch (new Eta) \\
Gas-phase chemistry & CBM-Z \\
\hline
\end{tabular}


Acknowledgements. This work is supported by the Korea Environmental Industry \& Technology Institute through the Eco Innovation Program (ARQ201204015), and partly by the National Research Foundation of Korea Grant (no. 2009-0083527) funded by the Korean government (MSIP). The third author acknowledges partial support from National Science Foundation Collaboration in Mathematical Geosciences grant 0930265 and from NASA Modeling, Analysis and Prediction (MAP) program grant NNX10AG92G.

Edited by: A. Sandu

\section{References}

Buehner, M.: Ensemble-derived stationary and flow-dependent background-error covariances, Q. J. R. Meteorol. Soc., 131, 1013-1043, 2005.

Buehner, M., Houtekamer, P. L., Charette, C., Mitchell, H. L., and He, B.: Intercomparison of variational data assimilation and the ensemble kalman filter for global deterministic NWP. Part I: Description and single-observation experiments, Mon. Weather Rev., 138, 1567-1586, 2010.

Constantinescu, E. M., Chai, T., Sandu, A., and Carmichael, G. R.: Autoregressive models of background errors for chemical data assimilation, J. Geophys. Res., 112, D12309, doi:10.1029/2006JD008103, 2007.

Eibern, H. and Schmidt, H.: A four-dimensional variational chemistry data assimilation scheme for Eulerian chemistry transport modeling, J. Geophys. Res., 104, 18583-18598, 1999.

Evensen, G.: The ensemble Kalman filter: theoretical formulation and practical implementation, Ocean Dynam., 53, 343-367, 2003.

Fast, J. D., Gustafson Jr., W. I., Easter, R. C., Zaveri, R. A., Barnard, J. C., Chapman, E. G., Grell, G. A., and Peckham, S. E.: Evolution of ozone, particulates, and aerosol direct radiative forcing in the vicinity of Houston using a fully coupled meteorology-chemistry-aerosol model, J. Geophys. Res., 111, D21305, doi:10.1029/2005JD006721, 2006.

Grell, G. A., Emeis, S., Stockwell, W. R., Schoenemeyer, T., Forkel, R., Michalakes, J., Knoche, R., and Seidl, W.: Application of a multiscale, coupled MM5/chemistry model to the complex terrain of the VTALP valley campaign, Atmos. Environ., 34, 1435$1453,2000$.
Grell, G. A., Peckham, S. E., Schmitz, R., McKeen, S. A., Frost, G., Skamarock, W. C., and Eder, B.: Fully coupled "online" chemistry within the WRF model, Atmos. Environ., 39, 6957-6975, 2005.

Houtekamer, P. L. and Mitchell, H. L.: Data assimilation using an ensemble Kalman filter technique, Mon. Weather Rev., 126, 796811, 1998.

Lahoz, W. A., Errera, Q., Swinbank, R., and Fonteyn, D.: Data assimilation of stratospheric constituents: a review, Atmos. Chem. Phys., 7, 5745-5773, doi:10.5194/acp-7-5745-2007, 2007.

Meena, G. S., Bhosale, C. S., and Jadhav, D. B.: Retrieval of stratospheric $\mathrm{O}_{3}$ and $\mathrm{NO}_{2}$ vertical profiles using zenith scattered light observations, J. Earth Syst. Sci., 115, 333-347, 2006.

Navon, I. M.: Data assimilation for numerical weather prediction: A review, in: Data Assimilation for Atmospheric, Oceanic and Hydrologic Applications, edited by: Park, S. K. and Xu, L., Springer, Berlin-Heidelberg, 21-65, 2009.

Park, S. K. and Zupanski, D.: Four-dimensional variational data assimilation for mesoscale and storm-scale applications, Meteorol. Atmos. Phys., 82, 173-208, 2003.

Singh, K., Jardak, M., Sandu, A., Bowman, K., Lee, M., and Jones, D.: Construction of non-diagonal background error covariance matrices for global chemical data assimilation, Geosci. Model Dev. Discuss., 3, 1783-1827, doi:10.5194/gmdd-3-1783-2010, 2010.

Thepaut, J.-N., Courtier, P., Belaud, G., and Lemaitre, G.: Dynamical structure functions in a four-dimensional variational assimilation: A case study, Q. J. R. Meteorol. Soc., 122, 535-561, 1996.

Wang, K.-Y., Lary, D. J., Shallcross, D. E., Hall, S. M., and Pyle, J. A.: A review on the use of the adjoint method in fourdimensional atmospheric-chemistry data assimilation, Q. J. R. Meteorol. Soc., 127, 2181-2204, 2001.

Whitaker, J. S., Compo, G. P., and Thepaut, J.-N.: A comparison of variational and ensemble-based data assimilation systems for reanalysis of sparse observations, Mon. Weather Rev., 137, 19911999, 2009.

Zupanski, M.: Maximum likelihood ensemble filter: Theoretical aspects, Mon. Weather Rev., 133, 1710-1726, 2005.

Zupanski, M.: Theoretical and practical issues of ensemble data assimilation in weather and climate, in: Data Assimilation for Atmospheric, Oceanic and Hydrologic Applications, edited by: Park, S. K. and Xu, L., Springer, Berlin-Heidelberg, 67-84, 2009. 